\title{
Proximal tubular dysfunction as an indicator of chronic graft dysfunction
}

\author{
N.O.S. Câmara ${ }^{1,2}$, W.W. Williams Jr. ${ }^{3}$ and A. Pacheco-Silva ${ }^{1,4}$ \\ ${ }^{1}$ Laboratório de Imunologia Clínica e Experimental, Disciplina de Nefrologia, Hospital do Rim e \\ Hipertensão, Escola Paulista de Medicina, Universidade Federal de São Paulo, São Paulo, SP, Brasil \\ ${ }^{2}$ Laboratório de Imunobiologia de Transplantes, Departamento de Imunologia, Instituto de Ciências \\ Biomédicas IV, Universidade de São Paulo, São Paulo, SP, Brasil \\ ${ }^{3}$ Massachusetts General Hospital, Harvard University, Boston, MA, USA \\ ${ }^{4}$ Unidade de Transplante Renal, Hospital Albert Einstein, Instituto Israelita de Ensino e Pesquisa, São \\ Paulo, SP, Brasil
}

Correspondence to: A. Pacheco-Silva, Disciplina de Nefrologia, EPM, UNIFESP, Rua Botucatu, 740, 04023-900 São Paulo, SP, Brasil

Fax: +55-11-5573-9652. E-mail: apacheco@nefro.epm.br

\begin{abstract}
New strategies are being devised to limit the impact of renal sclerosis on graft function. Individualization of immunosuppression, specifically the interruption of calcineurin-inhibitors has been tried in order to promote better graft survival once chronic graft dysfunction has been established. However, the long-term impact of these approaches is still not totally clear. Nevertheless, patients at higher risk for tubular atrophy and interstitial fibrosis (TA/IF) development should be carefully monitored for tubular function as well as glomerular performance. Since tubular-interstitial impairment is an early event in TA/IF pathogenesis and associated with graft function, it seems reasonable that strategies directed at assessing tubular structural integrity and function would yield important functional and prognostic data. The measurement of small proteins in urine such as $\alpha-1$-microglobulin, $\mathrm{N}$ acetyl-beta-D-glucosaminidase, alpha/pi S-glutathione transferases, $\beta$ - 2 microglobulin, and retinol binding protein is associated with proximal tubular cell dysfunction. Therefore, its straightforward assessment could provide a powerful tool in patient monitoring and ongoing clinical assessment of graft function, ultimately helping to facilitate longer patient and graft survival associated with good graft function.
\end{abstract}

Key words: Renal transplantation; Tubular proteins; Proximal tubular dysfunction; Chronic allograft nephropathy; Retinol binding protein

Research supported by a Roche Organ Transplantation Research Foundation grant and a CNPq productivity research grant to N.O.S. Câmara and A. Pacheco-Silva. Publication supported by FAPESP.

Received May 30, 2008. Accepted January 5, 2009

\section{Introduction}

Long-term kidney allograft survival is reduced by the unavoidable occurrence of tissue fibrosis, namely, glomerulosclerosis, tubular atrophy and interstitial fibrosis (TA/ IF). Since 1991 this condition has been known as chronic allograft nephropathy. However, chronic allograft nephropathy involves not only a chronic rejection diagnosis but also other entities that culminate with glomerulosclerosis and TA/IF such as chronic drug toxicity. The present article will consider a broader definition of this condition because many immune and non-immune factors are implicated in its pathogeny. Although much attention has been directed towards the individualization of immunosuppressive drugs once TA/IF has been diagnosed, little data are available showing the real long-term benefit of this strategy. Therefore, a more plausible approach to improve post-transplant graft survival would be the very early detection of TA/IF. Taking into consideration the physiological events that occur during development of graft fibrosis, we have pro- 
posed that early detection of proximal tubular cell dysfunction by urinary measurement of small proteins may offer a reliable and sensitive means of predicting long-term graft function. Here we review the concept underlying this hypothesis and presented the rationale for monitoring proximal tubular cell dysfunction in kidney allografts.

\section{Many roads to Rome: the undesirable natural history of chronic graft dysfunction}

In renal transplantation, the UNOS/OPTN registry data demonstrate a long-term graft loss rate of at least $40 \%$ at the 10-year follow-up time-point. This attrition is due mainly to two distinct conditions: death with a functioning graft and chronic graft dysfunction. These observations have been confirmed by recent analyses, where TA/IF stands as one of the most frequent causes of initiation of dialysis in the United States (1).

It is noteworthy that traditional outcome measures used to define allograft performance have been evolving over the past two decades. For example, in the pre-cyclosporine era, graft survival at one year was a benchmark measure. However, after the introduction of this calcineurin inhibitor $(\mathrm{Cl})$, there were marked improvements in 1year graft survival $(1,2)$. Subsequent to cyclosporine, in the 1990's, other milestones were reached, such as the rates of acute rejection (AR) to name but one example. Again, with the advent of potent, late-generation immunosuppressant agents - e.g., mycophenolate mofetil, tacrolimus, and induction therapy with antithymocyte agents - AR episodes eventually fell below a threshold of about 10 to $20 \%$ in most transplant centers (3). Therefore, AR events became outmoded as less robust measures of graft performance.

We are left, therefore, with a powerful paradox: despite improved immediate allograft performance as manifested by low rates of early acute rejection and excellent 1-year graft survival, long-term graft survival remains unacceptably compromised. Simply put: all renal allografts fail over time. This is largely due to TA/IF, which may be caused by long-term exposure to the lynchpins of immunosuppressive protocols, $\mathrm{Cl}$, viral infection (polyomavirus: BK virus), acute/subclinical rejection, ischemia and reperfusion injury, dyslipidemia/hypertension/diabetes, among others. To date, there are no reliable tests that can predict the development of chronic allograft dysfunction, while a genomic approach has been investigated in order to detect it. Because long-term graft decline has been the rule in kidney transplantation, there is a great need for the development of an index or marker that could be associated with long-term kidney allograft survival and performance.
It is broadly accepted today that TA/IF comprises distinct entities that are joined in a final common pathway due to the presence of tubulointerstitial fibrosis, glomerulosclerosis and vasculopathy (4). Nevertheless, the real contribution of drug toxicity, potentially hyperfiltration-mediated glomerulosclerosis resulting from nephron overload, and immune-mediated chronic rejection to tissue fibrosis is hardly measurable. Although, as stated earlier, the histological features of TA/IF can be seen in glomeruli, vessels and tubulointerstitium, its severity is graded mainly by the degree of TA and IF. The decrease in graft function in patients with graft fibrosis is mainly associated with the amount of TA/IF $(4,5)$.

Data from protocol biopsies suggest that the histological features of graft fibrosis can be detected early, even as early as 3 months post-transplantation; however, the negative impact on graft function may be evident in measurable terms only late in the course. Once significant fibrosis and associated TA/IF, as well as fall-out begin to affect renal function, the clinical presentation is variable, with patients presenting a range of clinical manifestations including proteinuria, hypertension, and renal insufficiency. Recently, Nankivell et al. (6) described the natural history of TA/IF, demonstrating that it develops during the first 6 months post-transplantation. These observations support the view that there are two phases in graft fibrosis development: the first phase is characterized by early tubulointerstitial damage, caused by ischemia/reperfusion injury and subclinical rejection and the later phase is chiefly characterized by microvascular and glomerular injury. Thus, TA/IF can be detected by protocol biopsies and its features correlated with long-term graft function. These sequential biopsy data also show that tubulointerstitial damage presages future renal sclerosis.

Our objective in this review, is to demonstrate the importance of tubule dysfunction in the development of graft fibrosis, and, most importantly, to show how this phenomenon can be exploited to monitor patients at high risk for developing the dysfunction.

\section{The epithelial-to-mesenchymal transition in renal tubular cells}

Ischemic and reperfusion injury (IRI) represents the earliest danger sign for tubular cells $(7,8)$. It has become increasingly clear that IRI not only promotes acute sublethal injury but also induces a chronic inflammatory cascade that ultimately leads to graft fibrosis. Early studies by Tilney et al. (9) have demonstrated persistent infiltration of macrophages and pro-inflammatory cytokines in isografts compared to sham-operated control animals. More re- 
cently, Burne-Taney et al. (10) demonstrated that increased myeloperoxidase activity and CD4+ T lymphocyte infiltration persist as late as 6 weeks after IRI, in association with substantial chemokine production. Furthermore, those kidneys exposed to IRI developed interstitial fibrosis.

The causal relationship between ischemia and tissue fibrosis came to light recently when several groups demonstrated that proximal tubular cells exposed to a variety of hypoxic conditions secrete chemokines that promote mononuclear infiltration. This early infiltration by macrophages, monocytes and $\mathrm{T}$ cells produces pro-fibrotic cytokines that activate other cells, including resident fibroblasts. Furthermore, injured proximal tubular cells can be differentiated into mesenchymal cells (11). During normal renal embryogenesis, mesenchymal-to-epithelial transition is obligatory for normal development. We have observed an inverse developmental transition associated with graft fibrosis, namely an epithelial-to-mesenchymal transition (EMT). EMT is a process whereby fully differentiated epithelial cells undergo transition to a mesenchymal phenotype giving rise to fibroblasts and myofibroblasts (12-14).

Experimentally, in renal fibrogenesis, it has been shown that some new fibroblasts are derived from tubular cells through EMT (11). The key cellular mediators of fibrosis are the myofibroblasts, which when activated serve as the primary collagen-producing cells. Myofibroblasts are generated from a variety of sources including resident mesenchymal cells, epithelial and endothelial cells, as well as from circulating fibroblast-like cells called fibrocytes that are derived from bone marrow stem cells (15). Much of the matrix is synthesized by interstitial myofibroblasts, recruited from resident fibroblasts and circulating precursors. In addition, a significant proportion is derived from EMT of tubule epithelial cells. The importance of EMT has been demonstrated in experimental models, where blockade of EMT attenuates renal fibrosis $(16,17)$.

Tubular EMT is an orchestrated, highly regulated process, involving loss of epithelial cell adhesion molecules and de novo mesenchymal-related molecule, such as vimentin or S100A expression. Many factors can initiate EMT, but transforming growth factor- $\beta$ (TGF- $\beta$ ) is the most potent. Signaling events in this pathway include activation of Smad/integrin-linked kinase and connective tissue growth factor (CTGF). Basement integrity is also a key regulator of EMT (18). During this process, the epithelial cells lose epithelial markers (cytokeratin, E-cadherin), apical-basal polarity and cell surface microvilli and acquire mesenchymal phenotype and fibrosing properties, as evidenced by the expression of vimentin, types I, III and IV collagen, and the collagen-synthesis chaperone protein, Hsp47. After injury, the detachment of the epithelial cells from the base- ment membrane leads to inhibition of type IV collagen assembly, probably by the $\alpha 1 \mathrm{NC} 1$ domain, and up-regulation of TGF- $\beta$. Up-regulation of matrix metalloproteases can also contribute to rupture of the tubular membrane, a critical event in EMT. These data agree with those previously described by Bonsib et al. (19). During this process, the epithelial tubular cells gain migratory properties and invade the surrounding tissues.

TGF- $\beta$ is believed to be a critical fibrogenic factor. In vitro, TGF- $\beta$ reversibly decreases expression of the epithelial markers, increases expression of mesenchymal ones, and plays a key role in the reorganization of actin fibers. TGF- $\beta$ can also modify the epithelial cell functionally, by down-regulating the megalin-cubilin-mediated endocytic apparatus. TGF- $\beta$ effectively suppresses E-cadherin expression in vitro. This means that epithelial cells will lose nearby cell contacts, structural integrity and cell polarity.

CTGF mediates the downstream pro-fibrotic and dysfunctional actions of TGF- $\beta$ and is highly expressed by tubular epithelial cells engulfed by interstitial fibrosis. There is increasing evidence suggesting that CTGF per se is involved in EMT. CTGF is a $38-\mathrm{kDa}$ heparin-binding cysteine-rich secreted extracellular matrix-associated protein involved in the synthesis of collagen, myofibroblast differentiation and cell proliferation, migration and adhesion (18). Recently, Liu et al. (16) demonstrated that proximal tubular cells cultivated in the presence of CTGF up-regulate alpha smooth muscle actin ( $\alpha$-SMA) and decrease Ecadherin expressions. In another study, Cheng et al. (20) determined the role of CTGF as a biomarker of kidney graft fibrosis. These investigators demonstrated that the CTGF was increased and up-regulated inside the graft, co-localized with $\alpha$-SMA in tubule epithelial cells, in a mouse model of kidney transplantation. Moreover, in vitro studies revealed that CTGF itself could induce EMT, independent of TGF- $\beta$. Finally, they demonstrated that high urinary levels of CTGF were present in patients with TA/IF and demonstrated good correlation with the histology. Taken together, these data demonstrated the potential of CTGF as a biomarker of TA/IF and possibly of renal fibrosis. Another growth factor, the tubular cell-derived, platelet-derived growth factor also promotes fibroblast proliferation (15).

Studying the role of EMT in vivo, Strutz et al. (21) demonstrated that tubular cells can express a fibroblast marker, fibroblast specific protein 1 (FSP-1) in an antimembrane glomerulonephritis model. FSP-1 has been associated with EMT and fibrosis. Rossini et al. (22) studied this marker in renal transplant biopsies. They found that FSP-1 was expressed more in the interstitium in cells with fibroblast-like morphology. Hertig et al. (23) studied 
the presence of EMT in biopsies of renal transplant patients 3 months after engraftment. In summary, they observed that in $41 \%$ of grafts, tubular epithelial cell exhibited mesenchymal transition markers, defined as vimentinpositive cells and cytoplasm positive staining for betacatenin, in the absence of acute graft dysfunction and advanced TA/IF. Their data suggest that EMT is an early phenomenon. Vongwiwatana et al. (24) demonstrated that tubular epithelial cells lose epithelial markers, such as Ecadherin, and present vimentin, S100A4 and $\alpha$-SMA in biopsies of patients with TA/IF. More importantly, these markers correlated with renal function.

The link between acute rejection and the development of fibrosis was partially confirmed by a study carried out by Robertson et al. (25). They demonstrated that S100A4, a marker of EMT, co-localized with CD8+ T cell infiltration, thus establishing a link between inflammation and EMT. Early on, the same group had demonstrated that TGF- $\beta$ could indeed stimulate the expression of CD103 $(\alpha E \beta 7$ integrin) in CD8+ T cells, the receptor of E-cadherin in tubular cells, upon which these cells can directly induce adjacent tubular epithelial cells to transform into proliferating fibroblasts that migrate across the tubular basement membrane, producing fibrotic lesions within the renal interstitium (26).

In the transplantation field, EMT can be induced by a wide range of injuries, such as immunosuppressive drugs (27), oxidative stress (28), acute rejection and, possibly, viral infection (29). Immunosuppressive drugs can induce or prevent EMT. Cyclosporine is associated with EMT in human proximal tubular cells via the TGF- $\beta / C T G F$ signaling pathway $(30,31)$. Recently, Copeland et al. (27) demonstrated that rapamycin and mycophenolate mofetil actually prevented and even reversed EMT in vitro.

It is noteworthy that EMT is a regulated process and proceeds mainly in a manner counteracting the physiologic effects of TGF- $\beta$. Experimental data show that bone morphogenic protein-7 (BMP-7) and hepatocyte growth factor can both reverse EMT in vitro and in vivo (32). BMP7, a 35-kDa homodimeric protein and a member of the TGF- $\beta$ superfamily, can preserve and even restore renal histology and function in different animal models of fibrosis. Therapeutic strategies that effect regression of renal fibrosis include angiotensin II inhibition, endothelin antagonism and BMP-7 treatment in various animal models of renal fibrosis.

Although these data have been derived primarily from experimental rodent models, human clinical data have also been generated that reveal a variety of potentially important strategies to slow the progress of graft fibrosis. There are increasing reports showing some improvement or preserva- tion of graft function following decrease or withdrawal of $\mathrm{Cl}$ and the addition of mycophenolate mofetil or rapamycin, and with strict control of dyslipidemia and hypertension. Nonetheless, it has become clear that the best way to "treat" graft fibrosis involves early diagnosis and intervention, before important key steps in tissue fibrosis and tubular atrophy take place. Because TA/IF primarily affects the tubulointerstitial compartment, one hypothesis is that it can be detected by measuring small tubular proteins such as $\alpha$-1-microglobulin, cleaved $\beta-2$ microglobulin, $\mathrm{N}$-acetyl-beta-D-glucosaminidase, alpha/pi S-glutathione transferase or retinolbinding protein (RBP) in urine.

\section{Tubular proteins as early markers of TA/IF development}

Tubular proteins such as $\alpha-1$-microglobulin, $\beta-2$ microglobulin, N-acetyl-beta-D-glucosaminidase, RBP and the brush border enzymes alanine-aminopeptidase and gamma-glutamyltransferase are small molecules (between 5 and $40 \mathrm{kDa}$ ) that are freely filtered by the glomeruli and virtually totally reabsorbed by the proximal tubular cells. They can be found at higher levels in urine when there is substantial filtration overload that surpasses tubular reabsorption capacity (Tm), or when there is tubular dysfunction allowing less back capture. Small molecular weight protein catabolism takes place in the proximal tubules mainly in the S1 and S2 segments of proximal tubules and less extensively in $\mathbf{S 3}$, where these constitute the most affected areas in ischemic/toxic injuries (33). Although poorly understood, it is known that tubular proteins are internalized by the megalin-cubilin-mediated endocytosis process. Megalin is a $600-\mathrm{kDa}$ transmembrane protein of the LDL receptor family with $\mathrm{NH}_{2}$ extracellular, single transmembrane and short intracytoplasmic domains. Megalin has been shown to endocytose pro-inflammatory and profibrotic cytokines as well as a variety of other important hormones and small proteins, including albumin, prolactin, insulin, vitamin D-binding protein, RBP, $\alpha$-1-microglobulin, and $\beta-2$ microglobulin (34). Another key protein, cubilin, also known as the intestinal intrinsic factor cobalamin receptor, is a $460-\mathrm{kDa}$ receptor with no transmembrane domain that requires assembly with megalin for its internalization. Cubilin is the receptor for albumin (together with megalin), transferrin, and immunoglobulin light chains, among others (34). Megalin-cubilin complexes are found in S1 and S2 portions inside the brush border. After ligation, the whole complex is endocytosed. Once inside the endosomes, the proteins are dissociated from their ligands, and directed to lysosomes for degradation. Megalin and cubilin return to the apical membrane surface (34). This 
endocytic process can be affected by immune or toxic factors. Furthermore, as stated earlier, TGF- $\beta$ has a negative effect on this process. Recent data on megalin-knockout mice, cubilin-deficient dogs and on patients with mutations in the cubilin gene have illustrated the importance of this mechanism.

It has been shown that tubular proteinuria is an earlier marker of proximal tubular dysfunction (PTD), comparable with urinary detection of phosphate, glucose, and amino acids. These factors have been extensively studied in nephrotoxicity related to heavy metals, glomerulonephritis and diabetic nephropathy (35). Indeed, it has been demonstrated that excretion of larger amounts of tubular proteins may precede glomerular involvement in diabetic nephropathy, even before the appearance of microalbuminuria. Specifically in glomerulonephritis, the presence of high levels of tubular protein in urine correlates with poor renal survival and steroid responsiveness (36-38). Thus, the presence of high urinary levels of small tubular proteins in a transplant recipient could be a silent marker of tubular dysfunction, which, if persistent, could herald loss of longterm graft function (as we will discuss below).

Much has been done in detecting tubular injury by using new biomarkers in renal transplant patients with acute rejection. Zhang et al. (39) investigated the specificity of kidney injury molecule-1 (Kim-1) as a marker of tubular injury in renal transplanted patients with acute rejection and in protocol biopsies. Kim-1 is a transmembrane tubular protein with extracellular immunoglobulin and mucin domains that so far has no identified functions. Its soluble ectodomain is released in urine after cleavage, being considered an excellent marker of proximal tubular injury induced by ischemic or toxic tubule stresses $(40,41)$. In their study, Kim-1 was expressed in proximal tubules in $28 \%$ of the protocol biopsies although none presented histological signs of tubular injury. More importantly, Kim1 expression correlated with functional outcome of the graft 18 months later. Similar data were obtained from those patients with acute rejection. These data demonstrated that a proximal tubular biomarker can be sufficiently sensitive to detect tubular injury, albeit during a rejection episode, and can also predict graft outcome (39). However, the authors did not measure protein in urine or any other low protein marker simultaneously with the immunohistochemistry to assure that this sensitivity was maintained or could prove useful for clinical surveillance.

Complementary to these data, van Timmeren et al. (42) did measure Kim-1 expression in urine and in biopsies of patients with different renal diseases, including glomerulonephritis. They observed that the biomarker correlated positively with renal damage and negatively with renal function. Moreover, Kim-1 co-localized with signs of inflammation and renal fibrosis. The potential of Kim-1 as a biomarker of proximal tubular injury was illustrated in several other models and in human studies of drug toxicity $(43,44)$, heavy metal nephropathy $(43,45)$, protein-overload nephropathy (46), and polycystic kidney disease (47).

RBP is the specific blood carrier protein for retinol and retinol acid, which are lipid-soluble vitamins. RBP is synthesized primarily by the liver, and then released into the blood, where it forms a complex with thyroxine-binding prealbumin and is distributed to various tissues. RBP plays a major role in storage and metabolism of vitamin A. Once RBP molecules are no longer able to interact effectively with prealbumin, they are excreted through the glomeruli, reabsorbed and degraded by proximal tubules. As a biomarker, the measurement of urinary RBP levels offers advantages over other proteins because its production is relatively constant. There is no known clinical situation in which overproduction could lead to abnormal urinary concentrations, and the protein is very stable across the whole range of urinary $\mathrm{pH}(37)$. Akin to $\mathrm{RBP}, \alpha-1$-microglobulin is a 27-kDa glycoprotein synthesized by liver cells involved in different roles in the body (bacterial adhesion to lithogenesis), and stable in acid urine. However, there is also evidence that it can be detected in glomerular proteinuria (48). There are commercially available kits for measuring these glycoproteins, facilitating their use in the out-patient clinical management of transplant recipients.

Recently, the urinary levels of RBP were shown to assist in detecting renal dysfunction in heart and kidney transplant patients, as early as 5 years before overt renal failure $(49,50)$. Specifically in heart transplant patients, we demonstrated that higher levels of RBP (>0.400 mg/L) implied a 3.87-fold risk of renal failure over 5 years of follow-up. In that study, 36 heart transplant recipients with a median age of 43.3 years were evaluated 15 months after surgery. All patients were under $\mathrm{Cl}$ therapy. Five patients of 13 with higher urinary levels of RBP developed end-stage renal disease that required dialysis. This renal function loss was mainly due to chronic cyclosporine toxicity. An even greater impact was seen in renal transplant recipients.

In 92 renal transplant patients with good and stable renal function at least 3 months after engraftment, 52\% were found to have increased urinary excretion of RBP. Ninetytwo patients were under $\mathrm{Cl}$ immunosuppression. During a five-year follow-up period, TA/IF was detected in 23 patients (25\%), 19 (82.6\%) of whom had high levels of urinary RBP. Five-year TA/IF-free and graft survivals were significantly worse in patients with higher levels of urinary RBP than in patients with normal levels ( 57.5 vs $89.9 \%, P=0.0004 ; 70.7$ vs $100 \%$, respectively, $\mathrm{P}=0.0002)$. The presence of high 
urinary RBP levels, namely the presence of PTD, was the strongest factor associated with the development of TA/IF $(\mathrm{RR}=5.3,95 \% \mathrm{Cl}=1.45-19.58, \mathrm{P}=0.012)$ compared to patients with normal urinary levels of RBP (50).

In agreement with these data, Teppo et al. $(51,52)$ have recently observed that $60 \%$ of patients with increased urinary excretion of $\alpha$-1-microglobulin at 6 months had renal function deterioration during a 4-year follow-up period. The authors measured 24-h urinary excretion of $\alpha-1$ microglobulin, albumin and TGF- $\beta$ in 79 renal transplant recipients with an average of 51 months post-transplantation. The urinary $\alpha-1$-microglobulin significantly correlated with urinary TGF- $\beta(r=0.508)$, with albumin $(r=0.220)$, and with the changes in 24-h creatinine clearance $(r=-0.273)$. More importantly, they observed that $60 \%$ of patients with an $\alpha$-1-microglobulin/creatinine ratio greater than $5 \mathrm{mg} /$ $\mathrm{mmol}$ presented a poor long-term outcome, namely renal function deterioration. It is worth to emphasize that they have initially shown that a sustained higher ratio of $\alpha-1$ microglobulin/creatinine measured in urine predicted AR in renal transplanted patients and correlated with the duration of cold ischemia $(51,52)$.

More recently, data from Nickerson's group (53) demonstrated a role for a specific form of $\beta-2$ microglobulin in graft monitoring. Although, as early described, $\beta-2$ microglobulin is unstable in low $\mathrm{pH}$ urine, this could become advantageous for patients with acute tubulointerstitial rejection. By using proteomic platform analyses, this group showed that a cleaved form of $\beta-2$ microglobulin (cleaved by aspartic proteases present in the urine of transplanted patients) was a good indicator of acute tubular injury (53).

There are two main reasons for the high levels of small tubular protein found in transplant recipients: i) high levels could be a marker of PTD, as a sign of distress of proximal tubular cells, or ii) they may be markers of definitive tubular damage, as a result of tubulointerstitial fibrosis before any important change in glomerular filtration has occurred. Our recent data support the former hypothesis. By analyzing 50 renal biopsies from patients with higher $(>1 \mathrm{mg} / \mathrm{L}, \mathrm{N}=$ 13) and normal $(N=37)$ urinary levels of RBP, we were able to demonstrate that PTD was associated with 2-year renal graft functional impairment. At various RBP measurements and from renal biopsy analysis, there was no expression of $\alpha$-SMA in proximal tubular cells of those patients with higher urinary levels of RBP (54).

\section{The near future of biomarkers}

Little is known about the molecular pathways underlying the histological features of TA/IF. Microarray is a powerful technique to detect thousands of genes related to these pathways (55). The identification of these putative genes is one further step towards the early diagnosis of TA/IF, and towards better management and prognosis of these patients (56). Mas et al. (56) used the microarray technique in kidney samples of transplanted patients with TA/IF in order to identify new noninvasive diagnostic markers. Comparing to normal kidneys, they found 728 differentially expressed genes related to fibrosis, extracellular matrix and inflammation. Moreover, the authors identified some genes in urine of patients with TA/IF compared to normal controls with stable renal function (18).

Proteomic techniques have been widely used in recent years for the discovery of new biomarkers or validation of established ones in different samples, urine, blood, and tissues. Using three different experimental models of drug toxicity, Bandara et al. (57) identified a rat-specific isoform of kininogen in the plasma of all three models. Interestingly, alpha-1 macroglobulin was up-regulated after cisplatin treatment and RBP was down-regulated in the plasma after Dserine therapy. Unfortunately, the group did not study these proteins in urine in order to certify their expression.

Proteomics is a growing field in transplantation due to the higher expectations of new biomarker discoveries in acute rejection and in TA/IF pathologies. Urinary proteomic analyses have already been shown to identify a specific profile in acute rejection with more than $90 \%$ sensitivity $(58,59)$. Its role in detecting proximal tubular dysfunction is totally undefined, although it has long been established that this condition alters the profile of urinary proteins compared to normal urinary proteome. An elegant study was performed by Cutillas et al. (60) in patients with Dent's disease, an X-linked recessive proximal tubular disorder due to mutations in the chloride/proton exchanger gene, CLCN5, in which the cubilin-megalin apparatus is ineffective. The authors identified a broad range of low molecular weight proteins, including RBP, $\beta-2$ glycoprotein-I and vitamin D-binding protein, which were differentially expressed, thus demonstrating the increasing potential of proteomic technology in detecting PTD.

In summary, although much effort is currently being directed toward preservation of renal allograft function once TA/IF has been detected, or preemptively, to avoid its occurrence by employing individualization of the immunosuppressive scheme (avoidance of $\mathrm{Cl}$ ), the ideal strategy by which the development of TA/IF could be eliminated altogether remains the missing link. We believe that enough information is available to recommend clinical trials that would help to modify drug regimes and clinical management accordingly. Small tubular proteins can easily be measured in urine and are highly reliable in predicting long-term graft function. 


\section{References}

1. Cecka JM. The UNOS Renal Transplant Registry. Clin Transpl 2002; 1-20.

2. Cecka JM. Comparing survival after transplantation with survival on dialysis: net benefit or a way to identify healthier patients? Transplantation 2006; 82: 619-620.

3. Meier-Kriesche HU, Schold JD, Srinivas TR, Kaplan B. Lack of improvement in renal allograft survival despite a marked decrease in acute rejection rates over the most recent era. Am J Transplant 2004; 4: 378-383.

4. Halloran PF, Melk A, Barth C. Rethinking chronic allograft nephropathy: the concept of accelerated senescence. J Am Soc Nephrol 1999; 10: 167-181.

5. Racusen LC, Halloran PF, Solez K. Banff 2003 meeting report: new diagnostic insights and standards. Am J Transplant 2004; 4: 1562-1566.

6. Nankivell BJ, Borrows RJ, Fung CL, O'Connell PJ, Allen RD, Chapman JR. The natural history of chronic allograft nephropathy. N Engl J Med 2003; 349: 2326-2333.

7. Chapman JR, O'Connell PJ, Nankivell BJ. Chronic renal allograft dysfunction. J Am Soc Nephrol 2005; 16: 30153026.

8. Banasik M, Klinger M. Chronic allograft nephropathy-immunologic and nonimmunologic factors. Ann Transplant 2006; 11: 7-10.

9. Takada M, Nadeau KC, Shaw GD, Marquette KA, Tilney $\mathrm{NL}$. The cytokine-adhesion molecule cascade in ischemia/ reperfusion injury of the rat kidney. Inhibition by a soluble Pselectin ligand. J Clin Invest 1997; 99: 2682-2690.

10. Burne-Taney MJ, Yokota N, Rabb H. Persistent renal and extrarenal immune changes after severe ischemic injury. Kidney Int 2005; 67: 1002-1009.

11. Liu Y. Epithelial to mesenchymal transition in renal fibrogenesis: pathologic significance, molecular mechanism, and therapeutic intervention. J Am Soc Nephrol 2004; 15: 1-12.

12. Bedi S, Vidyasagar A, Djamali A. Epithelial-to-mesenchymal transition and chronic allograft tubulointerstitial fibrosis. Transplant Rev 2008; 22: 1-5.

13. Li MX, Liu BC. Epithelial to mesenchymal transition in the progression of tubulointerstitial fibrosis. Chin Med J 2007; 120: 1925-1930.

14. Zeisberg M, Kalluri R. The role of epithelial-to-mesenchymal transition in renal fibrosis. J Mol Med 2004; 82: 175-181.

15. Wynn TA. Cellular and molecular mechanisms of fibrosis. $J$ Pathol 2008; 214: 199-210.

16. Liu BC, Li MX, Zhang JD, Liu XC, Zhang XL, Phillips AO. Inhibition of integrin-linked kinase via a siRNA expression plasmid attenuates connective tissue growth factor-induced human proximal tubular epithelial cells to mesenchymal transition. Am J Nephrol 2008; 28: 143-151.

17. Yoshikawa M, Hishikawa K, Marumo T, Fujita T. Inhibition of histone deacetylase activity suppresses epithelial-to-mesenchymal transition induced by TGF-beta1 in human renal epithelial cells. J Am Soc Nephrol 2007; 18: 58-65.

18. Burns WC, Kantharidis $P$, Thomas MC. The role of tubular epithelial-mesenchymal transition in progressive kidney disease. Cells Tissues Organs 2007; 185: 222-231.

19. Bonsib SM, Abul-Ezz SR, Ahmad I, Young SM, Ellis EN, Schneider DL, et al. Acute rejection-associated tubular basement membrane defects and chronic allograft nephrop- athy. Kidney Int 2000; 58: 2206-2214.

20. Cheng O, Thuillier R, Sampson E, Schultz G, Ruiz P, Zhang $\mathrm{X}$, et al. Connective tissue growth factor is a biomarker and mediator of kidney allograft fibrosis. Am J Transplant 2006; 6: 2292-2306.

21. Strutz F, Okada H, Lo CW, Danoff T, Carone RL, Tomaszewski JE, et al. Identification and characterization of a fibroblast marker: FSP1. J Cell Biol 1995; 130: 393-405.

22. Rossini M, Cheunsuchon B, Donnert E, Ma LJ, Thomas JW, Neilson EG, et al. Immunolocalization of fibroblast growth factor-1 (FGF-1), its receptor (FGFR-1), and fibroblast-specific protein-1 (FSP-1) in inflammatory renal disease. Kidney Int 2005; 68: 2621-2628.

23. Hertig A, Verine J, Mougenot B, Jouanneau C, Ouali N, Sebe $P$, et al. Risk factors for early epithelial to mesenchymal transition in renal grafts. Am J Transplant 2006; 6: 2937-2946.

24. Vongwiwatana A, Tasanarong A, Rayner DC, Melk A, HalIoran PF. Epithelial to mesenchymal transition during late deterioration of human kidney transplants: the role of tubular cells in fibrogenesis. Am J Transplant 2005; 5: 13671374.

25. Robertson $\mathrm{H}$, Ali $\mathrm{S}$, McDonnell BJ, Burt AD, Kirby JA. Chronic renal allograft dysfunction: the role of T cell-mediated tubular epithelial to mesenchymal cell transition. J Am Soc Nephrol 2004; 15: 390-397.

26. Robertson $H$, Wong WK, Talbot $D$, Burt AD, Kirby JA. Tubulitis after renal transplantation: demonstration of an association between CD103+ T cells, transforming growth factor beta1 expression and rejection grade. Transplantation 2001; 71: 306-313.

27. Copeland JW, Beaumont BW, Merrilees MJ, Pilmore HL. Epithelial-to-mesenchymal transition of human proximal tubular epithelial cells: effects of rapamycin, mycophenolate, cyclosporin, azathioprine, and methylprednisolone. Transplantation 2007; 83: 809-814.

28. Djamali A. Oxidative stress as a common pathway to chronic tubulointerstitial injury in kidney allografts. Am J Physiol Renal Physiol 2007; 293: F445-F455.

29. Horikawa T, Yang J, Kondo S, Yoshizaki T, Joab I, Furukawa $\mathrm{M}$, et al. Twist and epithelial-mesenchymal transition are induced by the EBV oncoprotein latent membrane protein 1 and are associated with metastatic nasopharyngeal carcinoma. Cancer Res 2007; 67: 1970-1978.

30. McMorrow T, Gaffney MM, Slattery C, Campbell E, Ryan MP. Cyclosporine A induced epithelial-mesenchymal transition in human renal proximal tubular epithelial cells. Nephrol Dial Transplant 2005; 20: 2215-2225.

31. Slattery C, Campbell E, McMorrow T, Ryan MP. Cyclosporine A-induced renal fibrosis: a role for epithelial-mesenchymal transition. Am J Pathol 2005; 167: 395-407.

32. Chatziantoniou C, Dussaule JC. Insights into the mechanisms of renal fibrosis: is it possible to achieve regression? Am J Physiol Renal Physiol 2005; 289: F227-F234.

33. Bernard A, Lauwerys R. Low-molecular-weight proteins as markers of organ toxicity with special reference to Clara cell protein. Toxicol Lett 1995; 77: 145-151.

34. Christensen El, Birn H. Megalin and cubilin: synergistic endocytic receptors in renal proximal tubule. Am J Physiol 
Renal Physiol 2001; 280: F562-F573.

35. D'Amico G, Bazzi C. Urinary protein and enzyme excretion as markers of tubular damage. Curr Opin Nephrol Hypertens 2003; 12: 639-643.

36. Sesso R, Santos AP, Nishida SK, Klag MJ, Carvalhaes JT, Ajzen $\mathrm{H}$, et al. Prediction of steroid responsiveness in the idiopathic nephrotic syndrome using urinary retinol-binding protein and beta-2-microglobulin. Ann Intern Med 1992; 116: 905-909.

37. Pereira AB, Nishida SK, Vieira JG, Lombardi MT, Silva MS, Ajzen $\mathrm{H}$, et al. Monoclonal antibody-based immunoenzymometric assays of retinol-binding protein. Clin Chem 1993; 39: 472-476.

38. Mastroianni KG, Nishida SK, Silva MS, Ajzen H, Pereira AB. Urinary retinol-binding protein as a prognostic marker in the treatment of nephrotic syndrome. Nephron 2000; 86: 109114.

39. Zhang PL, Rothblum LI, Han WK, Blasick TM, Potdar S, Bonventre JV. Kidney injury molecule-1 expression in transplant biopsies is a sensitive measure of cell injury. Kidney Int 2008; 73: 608-614.

40. Devarajan P. Emerging biomarkers of acute kidney injury. Contrib Nephrol 2007; 156: 203-212.

41. Han WK, Waikar SS, Johnson A, Betensky RA, Dent CL, Devarajan $\mathrm{P}$, et al. Urinary biomarkers in the early diagnosis of acute kidney injury. Kidney Int 2008; 73: 863-869.

42. van Timmeren MM, van den Heuvel MC, Bailly V, Bakker SJ, van Goor H, Stegeman CA. Tubular kidney injury molecule-1 (KIM-1) in human renal disease. J Pathol 2007; 212: 209-217.

43. Zhou Y, Vaidya VS, Brown RP, Zhang J, Rosenzweig BA, Thompson KL, et al. Comparison of kidney injury molecule1 and other nephrotoxicity biomarkers in urine and kidney following acute exposure to gentamicin, mercury, and chromium. Toxicol Sci 2008; 101: 159-170.

44. Espandiari P, Zhang J, Rosenzweig BA, Vaidya VS, Sun J, Schnackenberg $\mathrm{L}$, et al. The utility of a rodent model in detecting pediatric drug-induced nephrotoxicity. Toxicol Sci 2007; 99: 637-648.

45. Prozialeck WC, Vaidya VS, Liu J, Waalkes MP, Edwards $\mathrm{JR}$, Lamar PC, et al. Kidney injury molecule- 1 is an early biomarker of cadmium nephrotoxicity. Kidney Int 2007; 72 : 985-993.

46. van Timmeren MM, Bakker SJ, Vaidya VS, Bailly V, Schuurs TA, Damman J, et al. Tubular kidney injury molecule-1 in protein-overload nephropathy. Am J Physiol Renal Physiol 2006; 291: F456-F464.

47. Kuehn EW, Park KM, Somlo S, Bonventre JV. Kidney injury molecule-1 expression in murine polycystic kidney disease. Am J Physiol Renal Physiol 2002; 283: F1326-F1336.

48. Penders J, Delanghe JR. Alpha 1-microglobulin: clinical laboratory aspects and applications. Clin Chim Acta 2004;
346: 107-118.

49. Camara NO, Matos AC, Rodrigues DA, Pereira AB, Pacheco-Silva A. Urinary retinol binding protein is a good marker of progressive cyclosporine nephrotoxicity after heart transplant. Transplant Proc 2001; 33: 2129-2131.

50. Camara NO, Silva MS, Nishida S, Pereira AB, PachecoSilva A. Proximal tubular dysfunction is associated with chronic allograft nephropathy and decreased long-term renal-graft survival. Transplantation 2004; 78: 269-275.

51. Teppo AM, Honkanen E, Ahonen J, Gronhagen-Riska C. Changes of urinary alpha1-microglobulin in the assessment of prognosis in renal transplant recipients. Transplantation 2000; 70: 1154-1159.

52. Teppo AM, Honkanen E, Finne P, Tornroth T, GronhagenRiska C. Increased urinary excretion of alpha1-microglobulin at 6 months after transplantation is associated with urinary excretion of transforming growth factor-beta 1 and indicates poor long-term renal outcome. Transplantation 2004; 78: 719-724.

53. Schaub S, Wilkins JA, Antonovici M, Krokhin O, Weiler T, Rush D, et al. Proteomic-based identification of cleaved urinary beta2-microglobulin as a potential marker for acute tubular injury in renal allografts. Am J Transplant 2005; 5: 729-738.

54. Matos AC, Franco MF, Pacheco-Silva A. Proximal tubular dysfunction and vimentin expression as predictors of renal function in renal transplant. Am J Transplantation 2005; 5: 320 (Abstract)

55. Allison DB, Cui X, Page GP, Sabripour M. Microarray data analysis: from disarray to consolidation and consensus. Nat Rev Genet 2006; 7: 55-65.

56. Kong X, Mas V, Archer KJ. A non-parametric meta-analysis approach for combining independent microarray datasets: application using two microarray datasets pertaining to chronic allograft nephropathy. BMC Genomics 2008; 9: 98.

57. Bandara LR, Kelly MD, Lock EA, Kennedy S. A correlation between a proteomic evaluation and conventional measurements in the assessment of renal proximal tubular toxicity. Toxicol Sci 2003; 73: 195-206.

58. O'Riordan E, Orlova TN, Mei JJ, Butt K, Chander PM, Rahman S, et al. Bioinformatic analysis of the urine proteome of acute allograft rejection. J Am Soc Nephrol 2004; 15: 3240-3248.

59. Wittke S, Haubitz M, Walden M, Rohde F, Schwarz A, Mengel $\mathrm{M}$, et al. Detection of acute tubulointerstitial rejection by proteomic analysis of urinary samples in renal transplant recipients. Am J Transplant 2005; 5: 2479-2488.

60. Cutillas PR, Chalkley RJ, Hansen KC, Cramer R, Norden $A G$, Waterfield MD, et al. The urinary proteome in Fanconi syndrome implies specificity in the reabsorption of proteins by renal proximal tubule cells. Am J Physiol Renal Physiol 2004; 287: F353-F364. 